\title{
Measuring non-extensitivity parameters in a turbulent Couette-Taylor flow
}

\author{
Christian Beck \\ School of Mathematical Sciences, Queen Mary and Westfield College, \\ University of London, Mile End Road, London E1 4NS, UK

\section{Gregory S. Lewis} \\ Calimetrics, Inc., 815 Atlantic Ave., Suite 105, Alameda, California 94501, \\ USA

\section{Harry L. Swinney} \\ Center for Nonlinear Dynamics and Department of Physics, The University \\ of Texas at Austin, Austin, Texas 78712, USA
}

\begin{abstract}
We investigate probability density functions of velocity differences at different distances $r$ measured in a Couette-Taylor flow for a range of Reynolds numbers Re. There is good agreement with the predictions of a theoretical model based on non-extensive statistical mechanics (where the entropies are non-additive for independent subsystems). We extract the scale-dependent non-extensitivity parameter $q(r, R e)$ from the laboratory data.
\end{abstract}




\section{Introduction}

Recently a generalization of the ordinary formalism of statistical mechanics, so-called non-extensive statistical mechanics, has gained a lot of interest [1, 2, 3]. While in ordinary statistical mechanics the Boltzmann-Gibbs entropy is extremized (subject to constraints), in the generalized formalism the more general Tsallis entropies $S_{q}$ are extremized. Given probabilities $p_{i}$ of the microstates of the physical system under consideration, the Tsallis entropies are defined as

$$
S_{q}=\frac{1}{q-1}\left(1-\sum_{i} p_{i}^{q}\right)
$$

where the parameter $q$ is the non-extensitivity parameter. The Tsallis entropies are convex, take on their extremum for the uniform distribution, and preserve the Legendre transform structure of thermodynamics. However, in contrast to the Boltzmann-Gibbs entropy, they are non-extensive (nonadditive for independent subsystems) provided $q \neq 1$.

Ordinary statistical mechanics is contained as a special case in the generalized formalism, since in the limit $q \rightarrow 1$ the Tsallis entropies reduce to the Boltzmann-Gibbs entropy, $S_{1}=-\sum_{i} p_{i} \ln p_{i}$. Recent work indicates that the non-extensive formalism with $q \neq 1$ describes many systems, including those exhibiting Levy-type anomalous diffusion [4], particles produced in collider experiments near the Hagedorn phase transition [5, 6, 7], and various turbulent systems [8, 9, 10, 11]. A list of references on non-extensive statistical mechanics and its applications is given in [12]. Generally, it is not known how the parameter $q$ depends on the internal properties of the system under consideration.

In this paper we will apply the non-extensive formalism to turbulence. In [11], fully developed turbulent states were distinguished from spatio-temporal chaotic states that extremize the Tsallis entropies, and formulas were obtained for probability densities of longitudinal velocity differences measured at a distance $r$. Here we test the theoretical predictions by comparing them with experimental data obtained for turbulent Couette-Taylor flow for different Reynolds numbers Re and spatial scales $r$. For details on the experiment, see [13].

We will provide evidence that a slightly generalized version of the theory described in [11] yields a good fit to the experimentally measured proba-

bility densities. These fits were achieved by varying only one quantity, the 
non-extensitivity parameter $q$. We will also present the first systematic experimental results showing how $q$ depends on $R e$ and $r$.

The probability densities obtained from non-extensive statistical mechanics asymptotically decay with a power law with a rather large exponent $w$. In our model $w$ is related to $q$ by $w=(4-2 q) /(1-q)$. We will provide experimental evidence that there is a simple scaling law for the function $w(r, R e)$.

This paper is organized as follows. Section 2 describes the experiment. Section 3 summarizes the theory presented in [11], and the formalism is slightly generalized. Section 4 compares the experimentally measured probability densities with the theoretical predictions. Section 5 examines the dependence of the non-extensitivity parameter $q$ and the exponent $w$ on Reynolds number and the spatial scale.

\section{Experiment}

The experiments were conducted on a concentric cylinder system with the inner cylinder rotating and the outer cylinder at rest [13]. This CouetteTaylor system had a radius ratio of 0.724 . Measurements were made for Reynolds numbers $R e$ up to 540000, where $R e=\Omega a(b-a) / \nu$ ( $\Omega$ is the inner cylinder rotation rate, $a$ and $b$ are the inner and outer cylinder radii, and $\nu$ is the kinematic viscosity). With $R e=0$ initially, the flow exhibits a sequence of bifurcations with increasing $R e$. The last transition that has been observed occurs at $R e=13000$ [13]. Here we consider data for $R e \geq 69000$, where the flow is strongly turbulent.

Velocity measurements were made with a hot film probe located midway between the two cylinders. The rms velocity fluctuations were typically only $6 \%$ of the mean velocity; hence the data satisfy Taylor's frozen turbulence hypothesis, that is, temporal fluctuations recorded by the fixed velocity probe should accurately reflect the streamwise spatial fluctuations 13. 


\section{Theoretical model for probability densities of longitudinal velocity differences}

In [11] a perturbative approach to probability densities in fully developed turbulent flows was suggested, based on a small parameter $\sqrt{\gamma \tau}$, where $\tau$ is a typical time scale of the chaotic force driving the local velocity differences and $\gamma^{-1}$ is the relaxation time to the stationary state. From the experimentally observed skewness of turbulent distributions one can estimate the order of magnitude of the parameter $\sqrt{\gamma \tau}$ to be about 0.1 ; hence a perturbative approach makes sense. Assuming that longitudinal velocity differences, $u(r) \equiv v(x+r)-v(x)$, in fully developed turbulence extremize the Tsallis entropies, and that large classes of chaotic relaxation processes approach the Gaussian limit in a universal way (see [14]), one can obtain the following formulas [11,

$$
\begin{aligned}
p(u) & =\frac{1}{Z_{q}}(1+\beta(q-1) \epsilon(u))^{-\frac{1}{q-1}} \\
\epsilon(u) & =\frac{1}{2} u^{2}-c \sqrt{\gamma \tau}\left(u-\frac{1}{3} u^{3}\right)+O(\gamma \tau) \\
\beta & =\frac{2}{5-3 q},
\end{aligned}
$$

where $p(u)$ is the stationary probability density of velocity differences, and $\epsilon(u)$ is a (formal) effective energy associated with the velocity difference $u$. $\beta$ is a variance parameter that describes a (formal) inverse temperature in the non-extensive statistical mechanics. $Z_{q}$ is a normalization constant. $c$ is a non-universal constant, i.e., a constant that may be different for different experiments and that can also depend on $q$. However, the functional form $u-\frac{1}{3} u^{3}$ of the term of order $\sqrt{\gamma \tau}$ is expected to be universal (see [11, 14]). The parameter $q$ depends on the distance $r$ in an (a priori) unknown way.

For $c=0$ and $\beta=2 /(5-3 q)$, the density in eq. (2) has average value 0 and variance 1 . However, if $c \neq 0$ and $\beta$ is still $2 /(5-3 q)$, then the average of $\langle u\rangle$ is of order $\gamma \tau$ and the variance $\sigma=\left(\left\langle u^{2}\right\rangle-\langle u\rangle^{2}\right)^{1 / 2}$ is slightly different from 1 . An average precisely zero and a variance of unity are achieved with the rescaled (renormalized) distribution given by

$$
\tilde{p}(u)=\sigma p(\sigma(u-\langle u\rangle) .
$$


The term $\epsilon(u)$ in eq. (3) stands for an effective energy in the formalism of non-extensive statistical mechanics. Indeed, for $q \rightarrow 1$, the Boltzmann factor $p(u) \sim e^{-\beta \epsilon(u)}$ is recovered. Let us here slightly generalize the approach of [11] by considering more general effective energy levels given by

$$
\epsilon(u)=\frac{1}{2}|u|^{2 \alpha}-c \sqrt{\gamma \tau} \operatorname{sign}(u)\left(|u|^{\alpha}-\frac{1}{3}|u|^{3 \alpha}\right) .
$$

For $\alpha=1$, eq. (3) is recovered, but we will allow for more general exponents $\alpha$ as well. The physical idea behind this is similar to that of the $\beta$-model of turbulence [15], where only a certain fraction $\beta$ of the physical volume is considered to contain active eddies. In the $\beta$-model one essentially replaces the structure functions $\left\langle|u|^{m}\right\rangle$ by structure functions $\left\langle|u|^{\alpha m}\right\rangle$ with $\alpha \neq 1$ related to the intermittency parameter. Similarly, in our dynamical model we replace $|u|$ by $|u|^{\alpha}$. An exponent $\alpha$ slightly smaller than 1 may be interpreted as describing a fractal phase space and eddies that are not space-filling. The formalism of non-extensive statistical mechanics is designed to include such systems.

In the following we will see that while the choice $\alpha=1$ yields reasonably good fits of the experimental data, an exponent $\alpha$ slightly smaller than 1 yields the best fits to the data.

\section{Comparison with the experimental measure- ments}

We compare the probability density functions determined from the CouetteTaylor velocity measurements with the theoretical densities $\tilde{p}(u)$ obtained from non-extensive statistical mechanics. The result is shown in Fig. 1 for Reynolds number $R e=540000$, equivalent to a Taylor scale Reynolds number $R_{\lambda}=262$ [13]. The best fits were obtained by choosing $\alpha$ according to the empirical formula $\alpha=1-(q-1)=2-q$. For the strength of the skewness term we have chosen, $c \sqrt{\gamma \tau}=0.124(q-1)$. Then only one independent parameter $q$ is left, which is fitted for each experimentally measured distribution in such a way that the relative mean square deviation integrated over $u$ takes a minimum. Although we only vary a single parameter $q$, the agreement with the experimentally measured densities is excellent. If $\alpha$ is 
chosen as 1 (as originally suggested in [11]), the agreement is still reasonable but not as good as for $\alpha=2-q$.

Other theoretical approaches to turbulent densities [16]-22], based, for example, on stretched exponentials or other functional forms, usually fit only certain parts of the distribution (e.g., the tails), leaving other parts (e.g. the vicinity of the maximum) unaccounted for. Our formula yields good fits of the experimental data for the entire range of $u$-values. To demonstrate the good fit for all $r$ we present both a logarithmic plot, which emphasizes the tails (Fig. 1(a)), and a linear plot, which emphasizes the maximum and its vicinity (Fig. 1(b)). To the best of our knowledge, there is no other theoretical model that yields fits of similar quality. Figure 2 shows that the residual difference between theory and experiments for the same spatial scale, $r / \eta \approx 11$ (where $\eta$ is Kolmogorov scale [13]), decreases with increasing Re.

The function $q(r, R e)$, deduced from the experiment, will be examined in the next section. All relevant information on the densities appears to be encoded in this function, which is similar to an equation of state in ordinary thermodynamics.

\section{$5 \quad$ Measuring $q(r, R e)$}

We have determined the function $q(r, R e)$ for different spatial scales $r$ and Reynolds numbers $R e$ by minimizing the integrated relative quadratic deviation between measured distributions and theoretical distributions. For each Reynolds number, 38 different spatial scales were evaluated and the results are shown in Fig. 3. The non-extensitivity parameter $q$ varies with both the spatial scale $r / \eta$ and with the Reynolds number. There is a tendency to smaller $q$ for smaller Re. For small $r, q$ approaches the value $q_{0} \approx 1.185$. For large $r, q$ does not approach 1 but saturates at a slightly larger value, $q_{\infty} \approx 1.03$, the precise value being Reynolds number dependent. Hence small deviations from the Gaussian distribution remain at the largest scales. This may be a finite-system size effect.

The predicted probability densities for large $|u|$ exhibit power law decay, as can be deduced from eq. (2) and (6), where we neglect the term $O(\sqrt{\gamma \tau})$ (our formula for this term represents a perturbative result valid only for $|u|<1 / \sqrt{\gamma \tau} \approx 10)$ : 


$$
p(u) \sim|u|^{-w}
$$

with exponent $w$ given by

$$
w=\frac{2 \alpha}{q-1}=\frac{4-2 q}{q-1} .
$$

The exponent $w$ has the meaning that only moments $\left\langle|u|^{m}\right\rangle$ of the density with $m<(w-1)$ exist. This sounds like a severe restriction on the existence of structure functions, but since $w$ is rather large $(9<w<60$ for the CouetteTaylor data), this effect does not contradict the experimental measurements of structure functions. Fig. 4 shows the function $w(r, R e)$ determined from the experimental data. We observe that $w$ exhibits simple scaling behaviour for medium spatial scales,

$$
w(r)=4\left(\frac{r}{\eta}\right)^{\delta},
$$

where the exponent $\delta$ depends weakly on the Reynolds number Re. We find $\delta=0.440,0.395,0.360$, and 0.326 for $R e=69000,133000,266000$, and 540000 , respectively. For $r / \eta<10$, the experimental data saturate at $w \approx 9$. For $w>60$, fluctuations become large.

The constant in front of the experimentally determined power law is found to be $4.0 \pm 0.1$, independent of the Reynolds number. A simple argument for the value 4 could be as follows. Suppose we could measure the probability distribution of ideal turbulence in an unperturbed way down to the Kolmogorov scale $\eta$ for infinite Reynolds number. Assuming that the scaling law (9) remains valid for $R e \rightarrow \infty$ and $r \rightarrow \eta$ we obtain at $r=\eta$ the value $w(\eta)=4$, no matter what $\delta$ is. But for turbulence to make sense at least the third moment $\left\langle|u|^{3}\right\rangle$ should exist, since this is the most fundamental observable related to energy dissipation. Existence of the third moment at $r=\eta$ is guaranteed if $w=4+\epsilon$, where $\epsilon$ is an arbitrarily small positive number. This argument suggests that the constant should be 4 .

For $R e \rightarrow \infty$ the idealized small-scale turbulence at $r=\eta$ is characterized by the smallest possible $w$ where it makes sense to speak about energy dissipation (the third moment). Since $w=4$, the flatness factor $F=\left\langle u^{4}\right\rangle /\left\langle u^{2}\right\rangle^{2}$ as well as all higher moments at this scale would then diverge for $R e \rightarrow \infty$. This is compatible with experimental observation [23]. 


\section{References}

[1] C. Tsallis, J. Stat. Phys. 52, 479 (1988)

[2] C. Tsallis, R.S. Mendes and A.R. Plastino, Physica 261A, 534 (1998)

[3] C. Tsallis, Braz. J. Phys. 29, 1 (1999)

[4] C. Tsallis, S.V.F. Levy, A.M.C. Souza, R. Maynard, Phys. Rev. Lett. 75, 3589 (1995)

[5] I. Bediaga, E.M.F. Curado, J. Miranda, Physica 286A, 156 (2000)

[6] C. Beck, Physica 286A, 164 (2000)

[7] W.M. Alberico, A. Lavagno, P. Quarati, Eur. Phys. J. C12, 499 (2000)

[8] X.-P. Huang and C.F. Driscoll, Phys. Rev. Lett. 72, 2187 (1994)

[9] B.M. Boghosian, Phys. Rev. 53E, 4754 (1996)

[10] T. Arimitsu and N. Arimitsu, Phys. Rev. 61E, 3237 (2000)

[11] C. Beck, Physica 277A, 115 (2000)

[12] http://tsallis.cat.cbpf.br/biblio.htm

[13] G.S. Lewis and H.L. Swinney, Phys. Rev. 59E, 5457 (1999)

[14] A. Hilgers and C. Beck, Phys. Rev. 60E, 5385 (1999)

[15] U. Frisch, P.-L. Sulem, M. Nelkin, J. Fluid Mech. 87, 719 (1978)

[16] B. Castaing, Y. Gagne, E.J. Hopfinger, Physica 46D, 177 (1990)

[17] I. Hosokawa, Phys. Rev. 49E, 4775 (1994)

[18] C. Meneveau and K.R. Sreenivasan, J. Fluid Mech. 224, 429 (1991)

[19] A. Polyakov, Phys. Rev. 52E, 6183 (1995)

[20] S. Grossmann and D. Lohse, Eur. Phys. Lett. 21, 201 (1993)

[21] L.P. Wang, S. Chen, J.G. Brasseur, J.C. Wyngaard, J. Fluid Mech. 309, 113 (1996)

[22] C. Beck, Phys. Rev. 49E, 3641 (1994)

[23] K.R. Sreenivasan and R.A. Antonia, Annu. Rev. Fluid Mech. 29, 435 (1997) 


\section{Acknowledgement}

Part of this research was performed during C.B.'s stay at the Institute for Theoretical Physics, University of California at Santa Barbara, supported in part by the National Science Foundation under Grant No. PHY94-07194. C.B. also gratefully acknowledges support by a Leverhulme Trust Senior Research Fellowship of the Royal Society. The research by H.L.S. was supported by the U.S. Office of Naval Research.

\section{Figure captions}

Fig. 1. Experimentally measured probability density functions of the velocity differences for the Couette-Taylor experiment at $R e=540000$ are compared with the theoretical curves $\tilde{p}(u)$ (for $\alpha=2-q$ ): (a) Logarithmic plot, (b) Linear plot. The logarithmic plot is sensitive to the tails, while the linear plot is sensitive to the vicinity of the maximum. For the experimental curves, the distances $r / \eta$ (where $\eta$ is the Kolmogorov length scale) are, from top to bottom: 11.6, 23.1, 46.2, 92.5, 208, 399, 830, and 14400. For the theoretical curves, the values of the non-extensivity parameter $q$ are, from top to bottom: 1.168, 1.150, 1.124, 1.105, 1.084, 1.065, 1.055, and 1.038. For better visibility, each distribution in (a) is shifted by -1 unit along the $y$ axis, and each distribution in (b) is shifted by -0.1 unit along the $y$ axis.

Fig. 2. Relative difference $d(u) \equiv\left(p_{t h}(u)-p_{\text {expt }}(u)\right) / p_{t h}(u)$ between theoretical and experimental probability densities at $r / \eta=12$. The difference decreases with increasing Reynolds number $R e=69000(+), 133000(\times)$, $266000(*)$, and $540000(\square)$. For each Reynolds number the best possible fit is used ( $q=1.148,1.159,1.167$, and 1.168, respectively).

Fig. 3. The fitness parameter $q(r, R e)$ deduced from a least square fit of the velocity data to the theoretical probability density. The Reynolds numbers are $69000(+), 133000(\times), 266000(*)$, and $540000(\square)$.

Fig. 4. The exponent $w=(4-2 q) /(q-1)$ describing the decay rate of the probability density for large $|u|$. Scaling behavior of $w(r)$ is observed for a large range of distances $r$. The Reynolds numbers are $69000(+), 133000$ $(\times), 266000(*)$, and $540000(\square)$. The straight lines correspond to the power 
law $w(r)=4(r / \eta)^{\delta}$ with $\delta=0.440,0.395,0.360$, and 0.326 , respectively. 
Fig. 1a

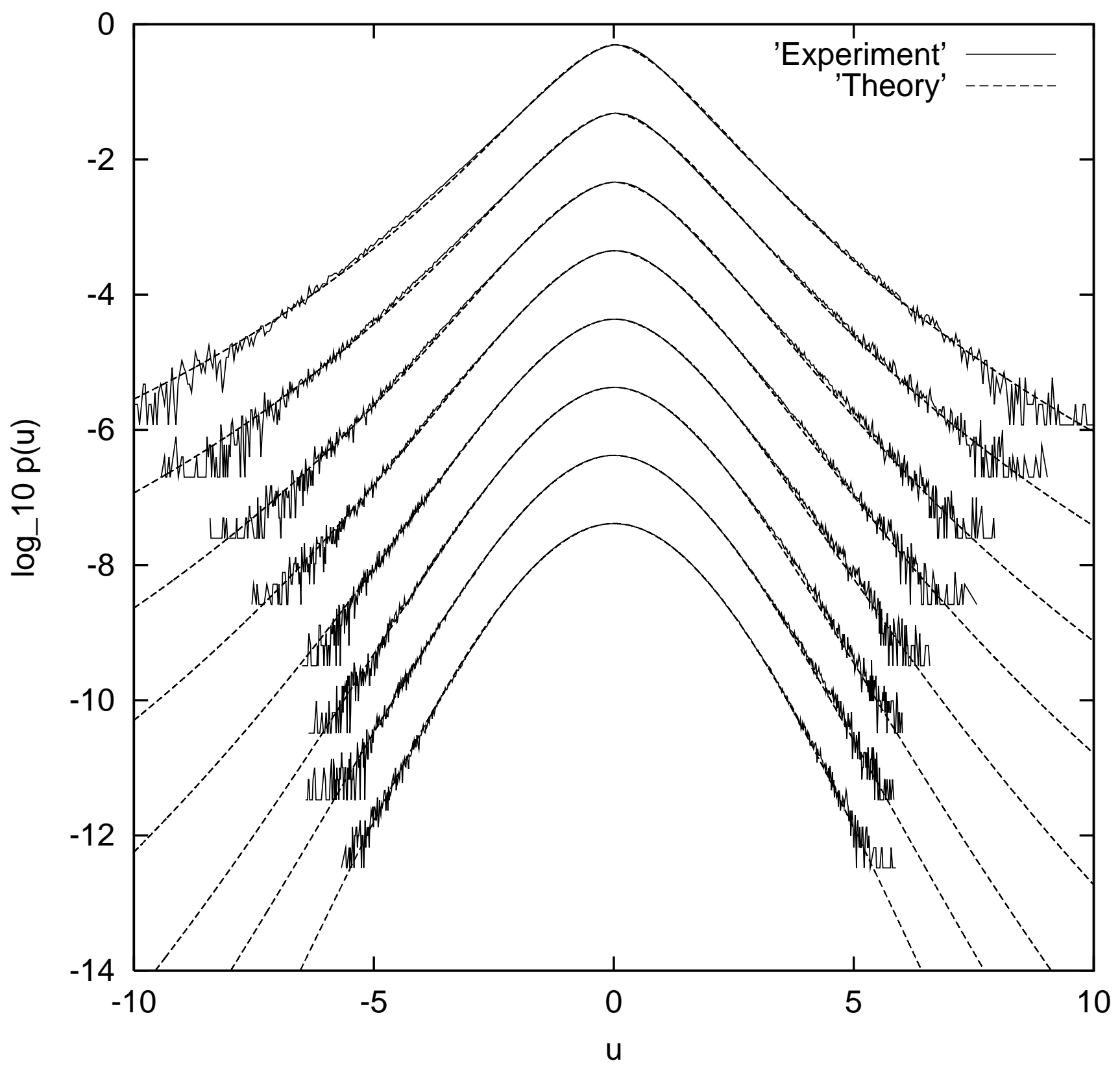


Fig. 1b

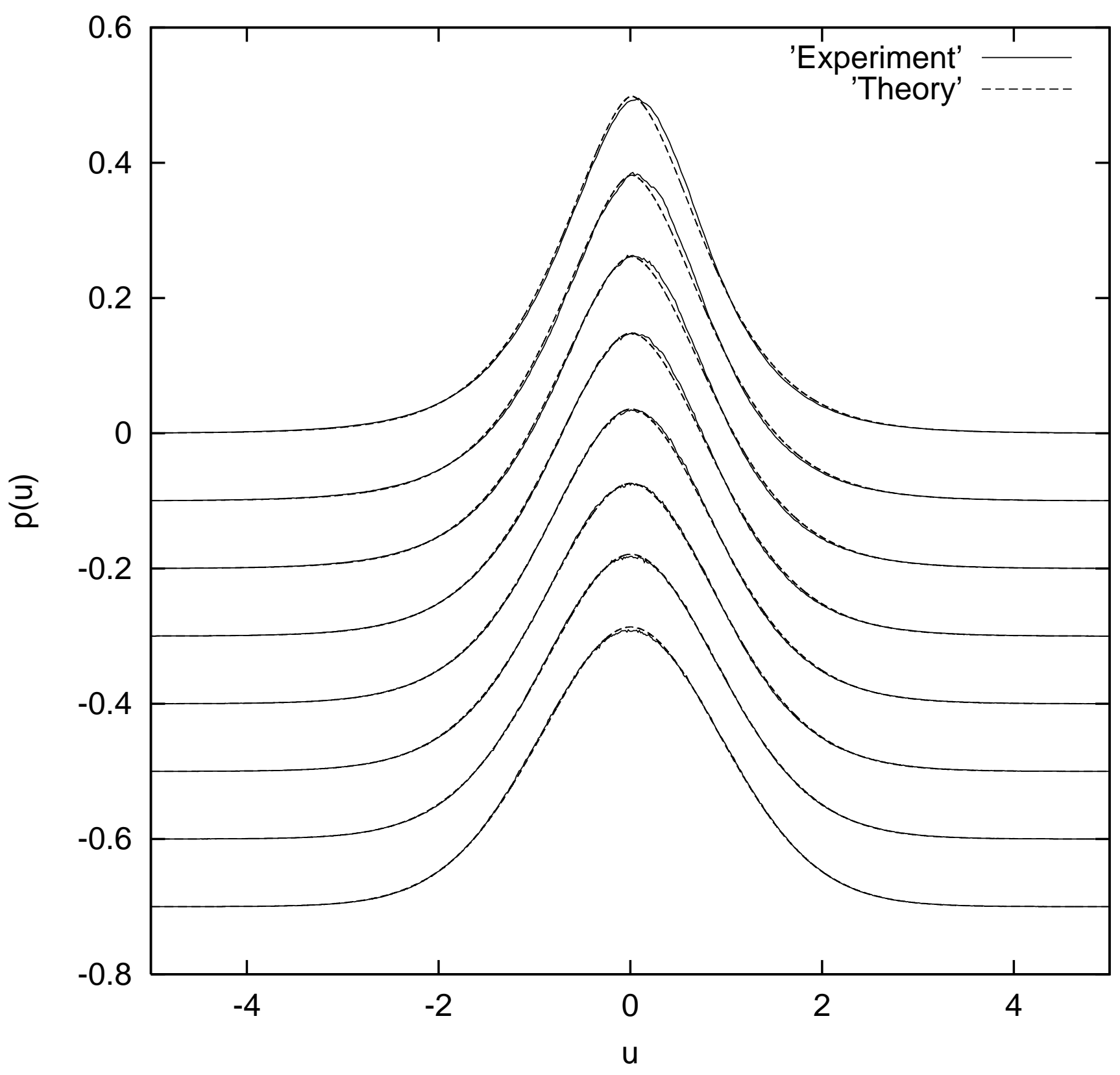


Fig. 2




Fig. 3

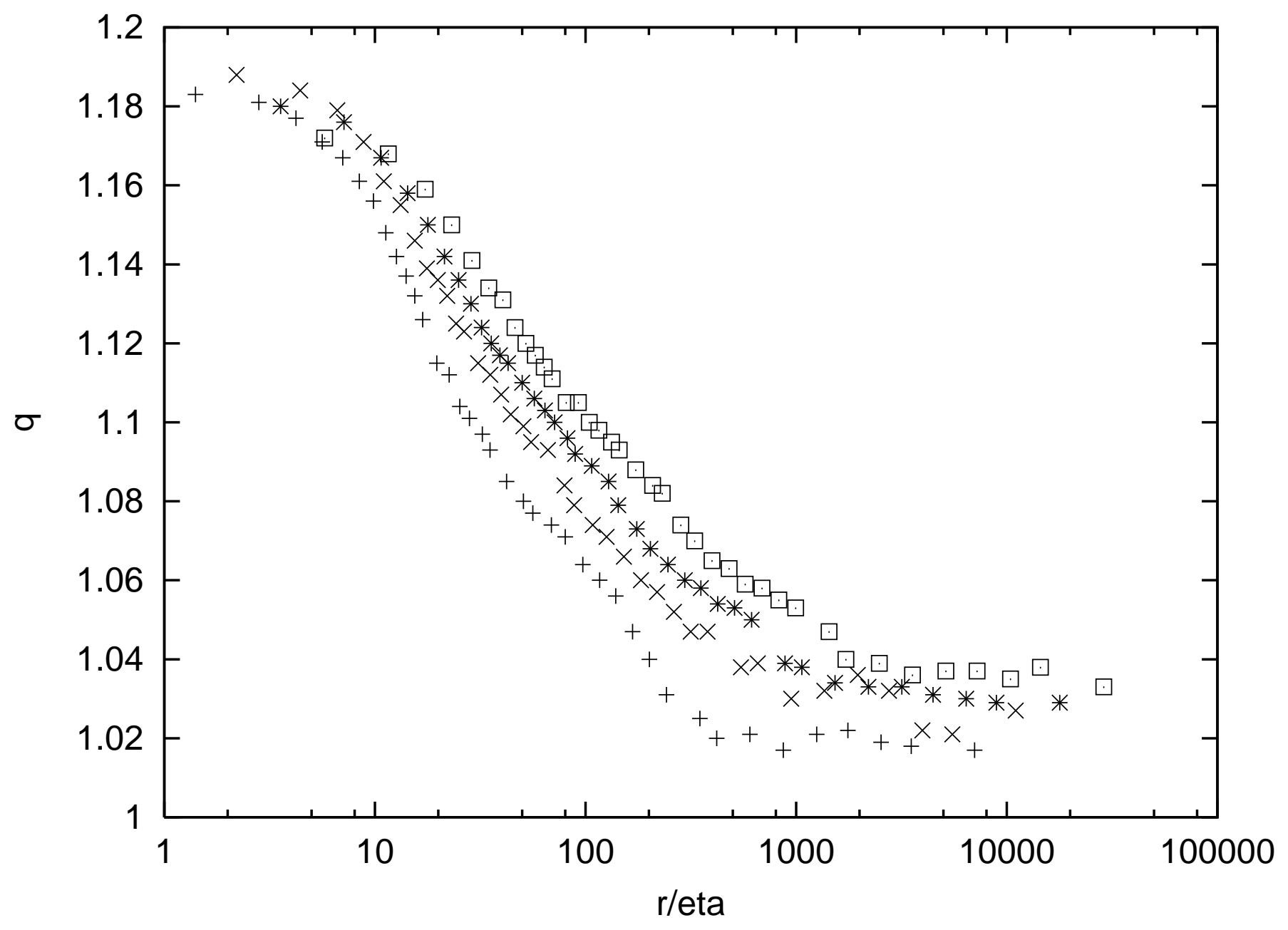


Fig. 4



